\title{
Molecular cloning and characterization of the glyceraldehyde-3-phosphate dehydrogenase gene from Penicillium expansum PE-12
}

\author{
T. Zhang ${ }^{1}$, Z. Qi ${ }^{2}$, Q.S. Yu ${ }^{1}$ and K.X. Tang ${ }^{1}$ \\ ${ }^{1}$ Plant Biotechnology Research Center, \\ School of Agriculture and Biology, \\ Shanghai Jiao Tong University, Shanghai, China \\ ${ }^{2}$ School of Life Science and Biotechnology, Shanghai Jiao Tong University, \\ Shanghai, China \\ Corresponding author: K.X. Tang \\ E-mail: kxtang@sjtu.edu.cn / kxtang1@yahoo.com
}

Genet. Mol. Res. 12 (3): 2442-2454 (2013)

Received June 24, 2012

Accepted August 31, 2012

Published February 6, 2013

DOI http://dx.doi.org/10.4238/2013.February.6.2

\begin{abstract}
Penicillium expansum produces large amounts of lipase, which is widely used in laundry detergent and leather industry. We isolated the glyceraldehyde-3-phosphate dehydrogenase gene $(P e G P D)$ from $P$. expansum PE-12 through reverse transcriptase PCR and 5'-3'rapid amplification of cDNA ends (RACE-PCR). The gene is $1266 \mathrm{bp}$ long, including an ORF of $1014 \mathrm{bp}$, encoding a polypeptide chain of 337 amino acids. A phylogenetic tree based on GPD proteins showed that $P$. expansum is close to Aspergillus species, but comparatively distant from $P$. marneffei. Southern blot results revealed a single copy of $P e G P D$, and expression analysis gave evidence of high expression levels. $P e G P D$ genes have potential for genetic engineering of $P$. expansum for industrial lipase production.
\end{abstract}

Key words: Glyceraldehyde-3-phosphate dehydrogenase gene;

Penicillium expansum; 5'-3'-RACE-PCR; Codon bias;

Homology modeling; Southern blot 


\section{INTRODUCTION}

Penicillium expansum is of considerable importance from a biotechnological aspect as a consequence of its content of versatile lipase (Dai and Xia, 2005). The lipase secreted by $P$. expansum (called PEL) can effectively hydrolyze the 3 ester bonds of triacylglycerol under alkaline condition (Bian et al., 2005) and is therefore widely used in laundry detergent and leather industry (www.leveking.com). However, back to the first discovery of PEL, the lipase productivity of this species was rather low, which made it impractical for commercial production (Stocklein et al., 1993). Later on, several strategies were adopted to maximize the production level of PEL including successive mutagenesis and selection (Zhang et al., 2001), fermentation condition optimization (Dai and Xia, 2005), and heterologous expression (Yuan et al., 2003; Zhang et al., 2010). After 2 decades of mutagenesis and selection accompanied by fermentation condition optimization, the industrial production of PEL was realized. However, due to excessive mutagenesis and selection, P. expansum mutants, such as the industrial strain $P$. expansum PE-12, became blunt to physical and chemical mutagens, which was a tremendous obstacle for further enhancement of its lipase productivity. Under such circumstances, reasonable genetic modification of the industrial strain based on homologous expression should be a promising strategy to resolve this problem.

However, to the best of our knowledge, no homologous gene promoters have been reported in P. expansum. Previous observations in yeast and higher eukaryotes have demonstrated that the glyceraldehyde-3-phosphate dehydrogenase (GPD, EC 1.2.1.12) gene (GPD) is under the control of a strong constitutive promoter. In such case, the expression level of the GPD protein could reach up to $5 \%$ of the soluble cellular protein (Piechaczyk et al., 1984). Furthermore, 2-5\% of the poly(A)+ RNA in yeast is GPD mRNA (Holland and Holland, 1978). Therefore, the promoters of GPD genes were widely used in homologous expression for strain improvements ( $\mathrm{Li}$ et al., 2000; Kajita et al., 2004).

As part of the effort to develop a homologous expression system for P. expansum PE12 based on a $G P D$ promoter, we report here for the first time, the cloning and characterization of an ORF, which encodes a GPD. Molecular organization, codon usage, copy number, and expression analysis are discussed.

\section{MATERIAL AND METHODS}

\section{Strains, media, and oligonucleotides}

P. expansum PE-12 is an industrial strain that has been subjected to several generations of mutagenesis to increase its lipase productivity. The strain was cultivated in fermentation medium containing $5 \mathrm{~g} / \mathrm{L}$ starch, $40 \mathrm{~g} / \mathrm{L}$ soybean powder, $2 \mathrm{~g} / \mathrm{L} \mathrm{Na}_{2} \mathrm{HPO}_{4}, 3 \mathrm{~g} / \mathrm{L} \mathrm{K} \mathrm{SO}_{4}$, $3 \mathrm{~g} / \mathrm{L} \mathrm{MgSO}_{4}, 5 \mathrm{~g} / \mathrm{L} \mathrm{CaCO}_{3}$, and $0.15 \mathrm{~g} / \mathrm{L} \mathrm{FeSO}_{4}$. Escherichia coli DH5a (Takara, Japan) was grown in LB medium (10 g/L tryptone, $5 \mathrm{~g} / \mathrm{L}$ yeast extract, $10 \mathrm{~g} / \mathrm{L}$ sodium chloride) and handled as described elsewhere (Sambrook and Russell, 2001).

Rapid amplification of cDNA end (RACE)-PCR oligonucleotides were designed using Primer premier 5.0, and all primers were synthesized by BBI (China). Molecular biological kits were used according to manufacturer manuals. 


\section{RNA isolation}

P. expansum PE-12 was grown in fermentation medium at $26^{\circ} \mathrm{C}$ for $72 \mathrm{~h}$ in an orbital shaker. The mycelia were collected by centrifugation, ground in liquid nitrogen, lysed with Trizol ${ }^{\mathbb{}}$ (Invitrogen, USA), deproteinated by chloroform and finally added to a silica column for RNA purification (Tiangen, China). After DNase treatment for 10 min, the RNA was eluted with RNase-free water.

\section{Cloning of the full-length cDNA of the PeGPD gene}

Cloning of PeGPD was by RACE primers GPD-F and GPD-R (Table 1), which were designed according to the conservative regions of the homologous GPD genes from GenBank. The partial conservative fragment of the $P e G P D$ gene was obtained by RT-PCR (AMV RT-PCR kit, Takara, Shiga, Japan) and subsequently used to design gene-specific primers (GPD3-1, GPD3-2, GPD5-1, and GPD5-2) for RACE. The cDNA was synthesized using the SMART technology (SMART ${ }^{\mathrm{TM}}$ RACE cDNA Amplification kit) for 3'- and 5'-RACE (Clontech Laboratories, Inc.). For 5'-RACE, RNA was reversely transcribed with the 5'-RACE CDS primer and SMART II oligonucleotide (provided in the kit). Cloning of $P e G P D$ was by 5'-RACE primers GPD5-1 and GPD5-2, which were designed according to the conserved ORF of the PeGPD gene. For 3'-RACE, RNA was reversely transcribed with the 3'-RACE CDS Primer A (provided in the kit). Based on the conserved fragment of the PeGPD gene, the specific primers GPD3-1 and GPD3-2 were designed and synthesized. The first round of PCR was carried out with primer GPD5-1 and Universal Primer A Mix (provided in the kit). The PCR product was then diluted 50-fold for subsequent amplification with primer GPD5-2 and Nested Universal Primer A (provided in the kit). 3'- and 5'-RACE was performed according to the SMART ${ }^{\mathrm{TM}}$ RACE cDNA Amplification Kit user manual. The full-length $P e G P D$ was deduced from the obtained sequences and subsequently amplified by proofreading RT-PCR amplification with primers GPDfull5 and GPDfull3. All the primers used in RACE are listed in Table 1.

Table 1. Primers used for PCR amplification in this study.

\begin{tabular}{lll}
\hline Primers & Purpose & Primer sequence (5' to 3') \\
\hline GPD-F & Clone & GTYGTCGCTGTCAACGACCCCY(C/T) \\
GPD-R & Clone & GACVAGCTTGATGAAGTTGGV(A/G/C) \\
GPD3-1 & 3'-RACE & GTGCTTCCTACGAGGAGATCAAG \\
GPD3-2 & 3'-RACE & CAAGGCCGCTTCCGAGAACGGCG \\
GPD5-1 & 5'-RACE & CATCCTTGGTGTAAGACTTGTTGTT \\
GPD5-2 & 5'-RACE & CCATGACGAACATGGGGGCATC \\
3'-RACE CDS primer A & RACE & AAGCAGTGGTATCAACGCAGAGTAC(T)30VN \\
5'-RACE CDS primer A & RACE & (T)25VNAAGCAGTGGTATCAACGCAGAGTACGCGGG \\
SMART II ${ }^{\text {TM A Oligo UPM }}$ & RACE & Long $(0.4$ M): CTAATACGACTCACTATAGGGCAAGCAGTGTATCAACGCAGAGT \\
& & Short (2 M): CTAATACGACTCACTATAGGGC \\
NUP & RACE & AAGCAGTGGTATCAACGCAGAGT \\
GPDfull5 & Clone & ATGGTTGTCCAGGTCGGTAT \\
GPDfull3 & Clone & CTACTGGCCATCGACACC \\
GPD-SouthF & Southern blot & TTCACCACTCAGGAGAAGGC \\
GPD-SouthR & Southern blot & CGATGCGGCAGGTAAGGT \\
GPDRT-F & RT-PCR & TATCAACGGTTCGGTCG \\
GPDRT-R & RT-PCR & GGATGGCAGCGGGGTCAC \\
PELRT-F & RT-PCR & CCCTTAACGCCTTCCCCA \\
PELRT-R & RT-PCR & AGCGTACATGCCATTGCC \\
\hline Y(C/T) V(A/G/C). & &
\end{tabular}

$\mathrm{Y}(\mathrm{C} / \mathrm{T}) \mathrm{V}(\mathrm{A} / \mathrm{G} / \mathrm{C})$. 


\section{Cloning of the genomic sequence}

Total genomic DNA was isolated from P. expansum PE-12 by the cetyltrimethylammonium bromide method and used as template in PCR amplification with primers, designed corresponding to the PeGPD full-length cDNA, GPDfull5 and GPDfull3 (Table 1) to check the existence of intron(s). The amplification reactions were denatured at $94^{\circ} \mathrm{C}$ for $5 \mathrm{~min}$, followed by 35 cycles of amplification $\left(94^{\circ} \mathrm{C}\right.$ for $30 \mathrm{~s}, 56^{\circ} \mathrm{C}$ for $30 \mathrm{~s}$, and $72^{\circ} \mathrm{C}$ for $\left.120 \mathrm{~s}\right)$ and ended after $72^{\circ} \mathrm{C}$ for $10 \mathrm{~min}$. The target band was recovered and cloned into pMD18-T vector, followed by sequencing.

\section{Bioinformatic analysis}

The percentage identities of the $P e G P D$ with other known $G P D$ genes were calculated using the BLAST program at the National Center for Biotechnology Information (NCBI, http:// www.ncbi.nlm.nih.gov/BLAST). The amino acid sequence deduction as well as the theoretical molecular weight and isoelectric point (pI) calculation were carried out at website (http:// www.expasy.org). The phylogenetic analysis of the PeGPD protein and GPDs from other species was performed through alignment with CLUSTAL X (1.81). An evolutionary tree from the fulllength amino acid sequence available from animals, plants and microorganisms was conducted by the neighbor-joining method (Saitou and Nei, 1987) using MEGA version 3.1 (Kumar et al., 2004). The codon usage frequency of the PeGPD gene was calculated with the Countcodon program of the NCBI Codon Usage Database (http://www.kazusa.or.jp/codon/).

Secondary structure of the PeGPD protein was predicted using the SOMPA server (http://npsa-pbil.ibcp.fr/cgi-bin/npsa_automat.pl?page=npsa_sopma.html). A homology model of the PeGPD was further developed to investigate its catalytic and $\mathrm{NAD}^{+}$-binding domain. A protein-protein BLAST search to PDB was performed using PeGPD for potential structural templates to develop the homology model. The template selected was the GPD crystal structure from Oryctolagus cuniculus (PDB: 1J0X_R), which had $73 \%$ sequence identity to the query sequence. The model was built using the alignment model program at the SWISSMODEL website (http://swissmodel.expasy.org/). The PeGPD model was superposed on the template, and the $\mathrm{C} \alpha$ and backbone root mean square deviation values were calculated using FATCAT (Ye and Godzik, 2003) for validation of the model. The model was further checked with a Ramachandran plot using PROCHECK (Laskowski et al., 1993).

\section{Southern blot analysis}

The copy number of the GPD gene in $P$. expansum PE-12 was determined as follows. Genomic DNA was digested with the restriction enzymes EcoRI, HindIII, KpnI, DraI, and BamHI. Digestion products were then separated on a 1.0\% agarose gel and transferred to a Hybond $\mathrm{T}^{\mathrm{TM}}-\mathrm{N}^{+}$membrane (Amersham Pharmacia, UK). The membrane was probed with a labeled DNA fragment containing the structural PeGPD gene according to the Amersham protocol. Probes were amplified with the primers GPD-SouthF and GPD-SouthR and subsequently labeled using the Alkphos Direct Labeling kit (Amersham). Hybridization was carried out with high stringency wash at $55^{\circ} \mathrm{C}$ and signal detection was performed using the CDP-Star detection system (Amersham), according to manufacturer instructions. 


\section{Gene expression analysis}

The value of $G P D$ promoter in engineering the industrial strain P. expansum PE-12 was determined by comparing the transcriptional strength of GPD and PEL through semiquantitative RT-PCR. The RNA sample was digested with DNase I (RNase-free) prior to use. Aliquots of $400 \mathrm{ng}$ total RNA were employed in the reverse transcriptase reaction using a random hexamer primer for the synthesis of first-strand cDNA. The amplification reactions of RT-PCR were performed with gene-specific primers GPDRT-F and GPDRT-R for GPD and primers PELRT-F and PELRT-R for PEL under the following conditions: pre-denaturation at $94^{\circ} \mathrm{C}$ for $5 \mathrm{~min}$, followed by 29 cycles of $30 \mathrm{~s}$ at $94^{\circ} \mathrm{C}, 30 \mathrm{~s}$ at $56^{\circ} \mathrm{C}$ and $30 \mathrm{~s}$ at $72^{\circ} \mathrm{C}$. RT-PCR images were captured by a UVP transilluminator.

\section{RESULTS AND DISCUSSION}

\section{Characterization of the full-length cDNA and genomic DNA sequences of $P e G P D$}

Using the degenerate primers GPD-F and GPD-R, a specific fragment of about 850 bp was obtained, and the sequence alignment displayed a high similarity to GPDs from other fungi. Based on the sequence of this fragment, 2 pairs of primers were designed for 5'- and 3'-RACE. Following the steps described, the full-length cDNA of the PeGPD gene (GenBank accession No. JN389439) was obtained, which was subsequently verified by PCR and sequencing. This new $P e G P D$ gene was 1306 bp long comprising a 126-bp 5'-untranslated region (UTR), 1014-bp ORF encoding a 337-amino acid residue protein, and a 166-bp 3'-UTR (Figure 1). The 5'-UTR contains one putative TATA box and 3 CAAT boxes. A pyrimidinerich stretch was found at $-69 \mathrm{bp}$ upstream of the start codon. In a number of filamentous fungi, transcription initiation sites appear in or immediately downstream of such pyrimidine-rich sequence (Vastag et al., 2004). In the terminator region, a putative polyadenylation signal (ATTAA) was identified about $122 \mathrm{bp}$ downstream of the stop codon.

The PCR for genomic sequence resulted in a specific fragment of $1558 \mathrm{bp}$, which was $252 \mathrm{bp}$ longer than that of the coding sequence. Compared to the cDNA, the genomic gene contained four introns. The lengths of the 4 introns were 56, 70, 64, and 62 bp (Figure 2). This result coincided well with the exon-intron arrangement of $G P D$ genes from Curvularia lunata and Cochliobolus heterostrophus, both of which also showed a similar genomic pattern (Thirach et al., 2008).

\section{Codon usage in PeGPD}

The codon bias in GPD has been previously reported in Ganoderma lucidum (Fei et al., 2006), Rhizomucor miehei (Vastag et al., 2004), and Ermothecium ashbyi (Sengupta and Chandra, 2011). In this research, we also noticed a strong codon bias which may be related to the abundance of the corresponding tRNA molecules (Table 2). In PeGPD only 35 of 61 possible sense codons have been utilized, and moreover, only 30 of these 35 codons have been used more than once. According to previous research, this bias could suggest a strong promoter activity and high expression level (Van Bogaert et al., 2008). Several amino acids exhibit a strict preference for a single codon such as Phe (TTC), His (CAC), Lys (AAG), Glu 
(GAG), and Cys (TGC). When a choice among A, T, C, and G in the wobble position exists, there is a strong preference for $\mathrm{C}$. Codon bias revealed by this study might benefit downstream research on over-expression of valuable protein, such as PEL, in Penicillium hosts, because it provides a reference for the codon engineering of the target protein (Gustafsson et al., 2004).

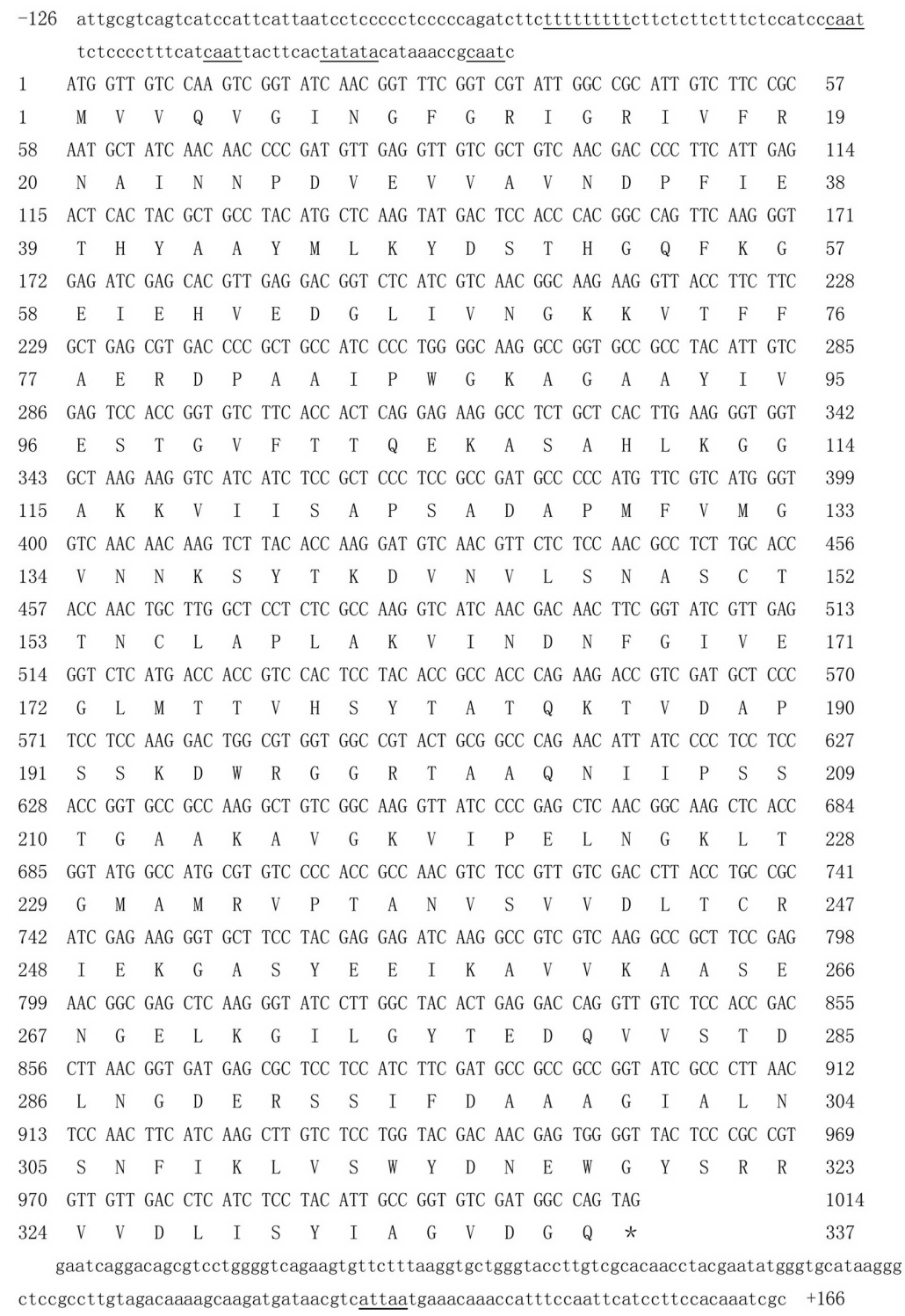

Figure 1. Nucleotide and predicted peptide sequence of the $P e G P D$ gene. ORF is shown in upper-case letters; 3'and 5'-UTRs are in lower-case letters. The predicted amino acids are displayed with single-letter symbols below their capitalized codon using DNAsmac2.0.2.6. Putative CAAT boxes, TATA box with flanking pyrimidine-rich stretch in 5'-UTR and polyadenylation sequence in 3'-UTR are underlined. This sequence is available in GenBank database under the accession No. JN389439. 


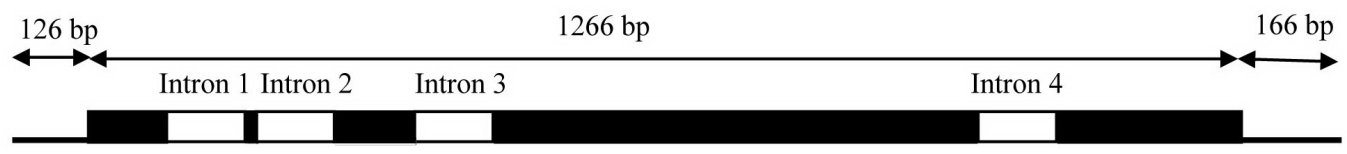

Figure 2. Gene structure of cloned $P e G P D$ (positions 1-1558 bp) (GenBank accession No. JN389439.). UTRs, exons, and introns are indicated by bold lines, filled boxes, and open boxes, respectively.

\begin{tabular}{|c|c|c|c|c|c|c|c|}
\hline Codon & Amino acid & GPD1 & GPD2 & Codon & Amino acid & GPD1 & GPD2 \\
\hline TTT & Phe & 0 & 0 & TAT & Tyr & 0.09 & 1 \\
\hline TTC & Phe & 1 & 11 & TAC & Tyr & 0.91 & 10 \\
\hline TTA & Leu & 0 & 0 & TAA & - & 0 & 0 \\
\hline TTG & Leu & 0.13 & 2 & TAG & - & 1 & 1 \\
\hline CTT & Leu & 0.31 & 5 & CAT & His & 0 & 0 \\
\hline СТC & Leu & 0.56 & 9 & CAC & His & 1 & 5 \\
\hline CTA & Leu & 0 & 0 & CAA & Gln & 0.14 & 1 \\
\hline CTG & Leu & 0 & 0 & CAG & Gln & 0.86 & 6 \\
\hline ATT & Ile & 0.25 & 6 & AAT & Asn & 0.05 & 1 \\
\hline ATC & Ile & 0.75 & 18 & AAC & Asn & 0.95 & 20 \\
\hline ATA & Ile & 0 & 0 & AAA & Lys & 0 & 0 \\
\hline ATG & Met & 1 & 7 & AAG & Lys & 1 & 22 \\
\hline GTT & Val & 0.33 & 12 & GAT & Asp & 0.39 & 7 \\
\hline GTC & Val & 0.67 & 24 & GAC & Asp & 0.61 & 11 \\
\hline GTA & Val & 0 & 0 & GAA & Glu & 0 & 0 \\
\hline GTG & Val & 0 & 0 & GAG & Glu & 1 & 18 \\
\hline TCT & Ser & 0.13 & 3 & TGT & Cys & 0 & 0 \\
\hline TCC & Ser & 0.87 & 20 & TGC & Cys & 1 & 3 \\
\hline TCA & Ser & 0 & 0 & TGA & - & 0 & 0 \\
\hline TCG & Ser & 0 & 0 & TGG & Trp & 1 & 4 \\
\hline $\mathrm{CCT}$ & Pro & 0.09 & 1 & CGT & Arg & 0.55 & 6 \\
\hline $\mathrm{CCC}$ & Pro & 0.91 & 10 & CGC & Arg & 0.45 & 5 \\
\hline CCA & Pro & 0 & 0 & CGA & Arg & 0 & 0 \\
\hline CCG & Pro & 0 & 0 & CGG & Arg & 0 & 0 \\
\hline ACT & Thr & 0.19 & 4 & AGT & Ser & 0 & 0 \\
\hline $\mathrm{ACC}$ & Thr & 0.81 & 17 & AGC & Ser & 0 & 0 \\
\hline $\mathrm{ACA}$ & Thr & 0 & 0 & $\mathrm{AGA}$ & Arg & 0 & 0 \\
\hline ACG & Thr & 0 & 0 & AGG & Arg & 0 & 0 \\
\hline GCT & Ala & 0.35 & 13 & GGT & Gly & 0.68 & 21 \\
\hline GCC & Ala & 0.62 & 23 & GGC & Gly & 0.32 & 10 \\
\hline GCA & Ala & 0 & 0 & GGA & Gly & 0 & 0 \\
\hline GCG & Ala & 0.03 & 1 & GGG & Gly & 0 & 0 \\
\hline
\end{tabular}

Column GPD1 presents the fraction of synonymous codon. Column GPD2 gives the total number of specific codon in the $P e G P D$ gene.

\section{Characterization of the deduced PeGPD protein}

Calculated by the $\mathrm{pI} / \mathrm{MW}$ tool software (http://www.expasy.org), the $\mathrm{pI}$ and molecular weight of the PeGPD protein were presumed to be 6.01 and $35.97 \mathrm{kDa}$, respectively. The homology of the PeGPD protein was aligned with GPDs from animal, plant, bacterium, and fungi (Figure 3 ) and ranged from a minimum of $68 \%$ to a maximum of $87 \%$ with fungal GPD. Compared to GPDs from Aspergillus niger and P. canescens, PeGPD exhibited a high sequence identity of 87 and $86 \%$, respectively. Aligned with members of other phyla, sequence identity was still higher than $68 \%$, which displayed a considerable conservation among species. This result demonstrates that the protein obtained here belongs to that of GPD, which is well conserved, and shows a close evolutionary relationship in the central metabolic pathway. 
1

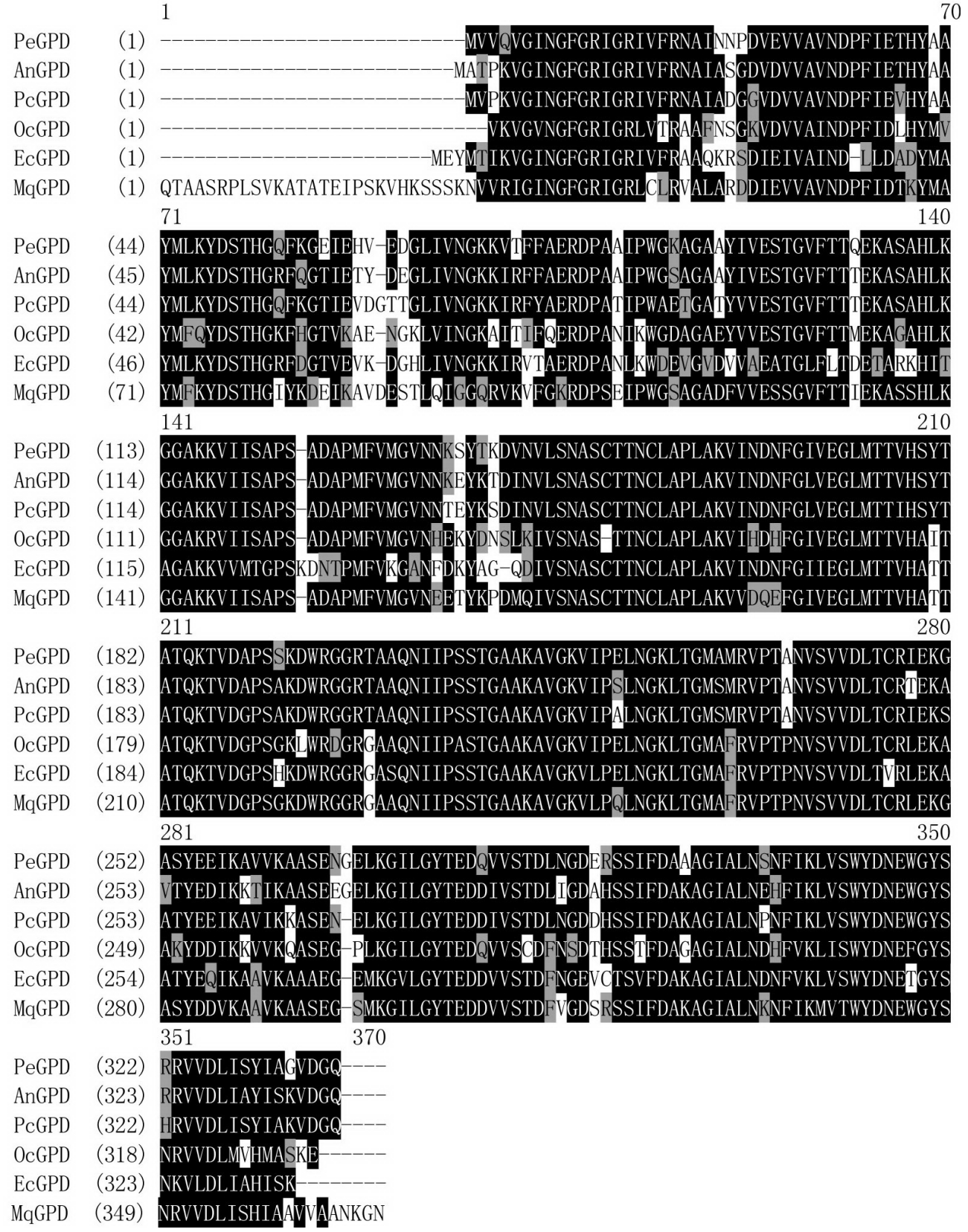

Figure 3. Mutiple sequence alignment of the $P e G P D$ with other known $G P D$ sequences using the CLUSTAL X software (1.81). The aligned GPDs are from Aspergillus niger (AnGPD, XP_001397496), Penicillium canescens (PcGPD, ACX54281), Oryctolagus cuniculus (OcGPD, 1J0X_R), Escherichia coli (EcGPD, NP_754078), and Marsilea quadrifolia (MqGPD, CAA06030). Highly conserved residues in all the sequences are indicated in white with black background and only partially conserved residues in the GPD sequences are shown in black with gray background. 


\section{Molecular evolution analysis}

A phylogenetic tree of GPD proteins from different organisms was drawn using MEGA version 3.1, which demonstrated that GPD proteins originated from a common ancestor and later diverged into several groups (Figure 4). Interestingly, phylogenetic analysis based on the GPD proteins suggested that, in terms of evolutionary distance, $P$. expansum was closer to Aspergillus species but comparatively distant from $P$. marneffei, which is consistent with previous morphological observations (Malloch, 1985). Similarly, this closer relationship to Aspergillus was also reported in P. chrysogenum based on the whole genome alignment (van den Berg et al., 2008), which may indicate that the Penicillium species evolved from different ancestors.

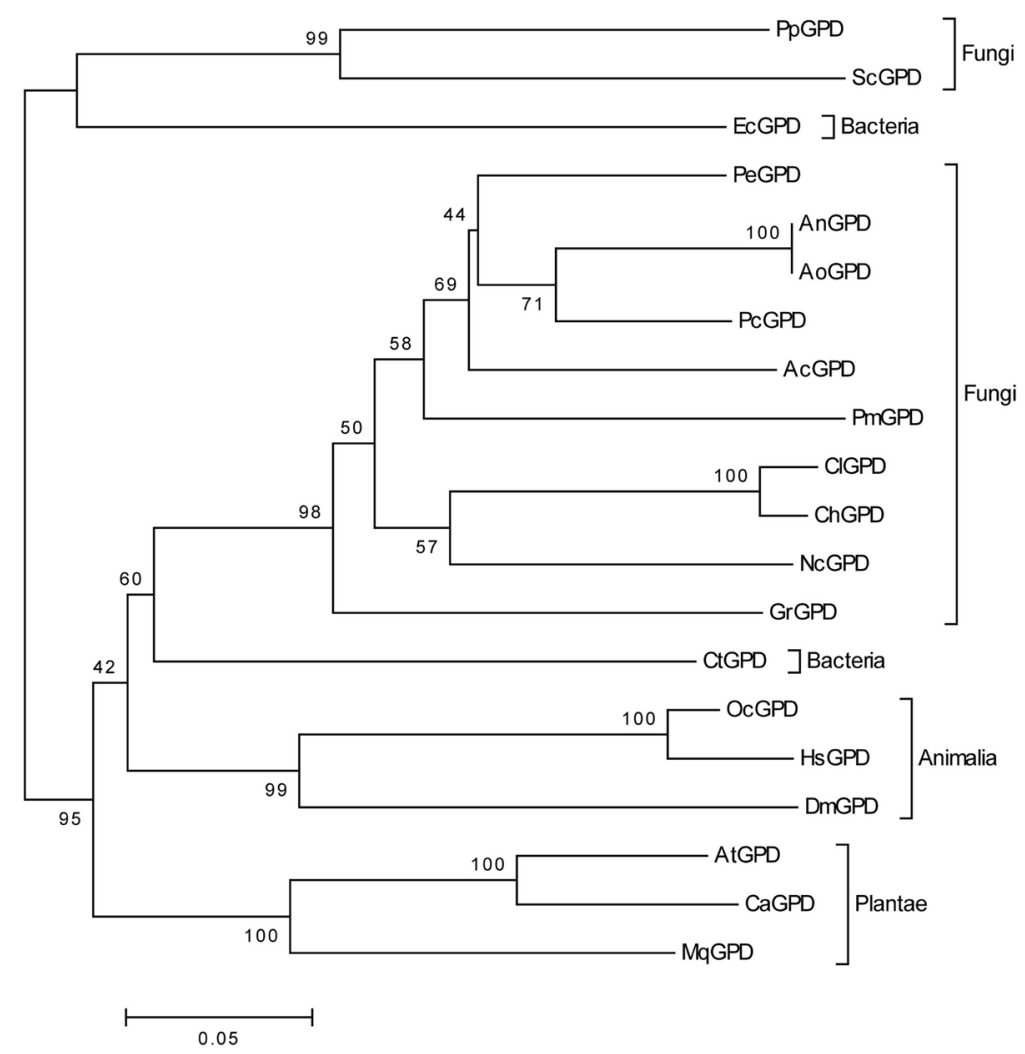

Figure 4. Phylogenetic tree showing the relationships between animal, plant, bacterial, and fungal GPD sequences. The MEGA version 3.1 software was used to construct the phylogenetic tree by neighbor-joining method. The numbers on the branches represent bootstrap support for 1000 replicates. The GPDs used in the phylogenetic tree analysis were from Aspergillus niger (AnGPD, XP_001397496), A. oryzae (AoGPD, XP_003189631), Penicillium canescens (PcGPD, ACX54281), Ajellomyces capsulatus (AcGPD, AF273703_1), P. marneffei (PmGPD, XP_002151104), Curvularia lunata (ClGPD, X58718), Cochliobolus heterostrophus (ChGPD, X63516), Neurospora crassa (NcGPD, U67457), Galiella rufa (GrGPD, AAT76626), Pichia pastoris (PpGPD, AAC49649), Saccharomyces cerevisiae (ScGPD, P00360), Oryctolagus cuniculus (OcGPD, 1J0X_R), Homo sapiens (HsGPD, NP_002037), Drosophila melanogaster (DmGPD, AAN09371), Arabidopsis thatiana (AtGPD, AAM67077), Capsicum annuum (CaGPD, CAC80377), Clostridium thermocellum (CtGPD, YP_001036571), Escherichia coli (EcGPD, NP_754078), and Marsilea quadrifolia (MqGPD, CAA06030). 


\section{Secondary structure prediction and homology modeling of PeGPD}

Secondary structure of the PeGPD protein was predicted using the SOMPA program, which displayed $\alpha$-helix (33.23\%), extended strand (23.74\%), $\beta$-turn (9.79\%), and random coil $(33.23 \%)$. The $\alpha$-helix and random coil constituted interlaced domination of the main part of the secondary structure.

The homology model of the PeGPD (Figure 5A) was developed with the closest PDB model 1J0X_R of $O$. cuniculus with a $73 \%$ sequence identity to the query sequence. Usually, the sequence alignment algorithms, which are critical for the quality of 3-D models, do not make gross errors when the percentage of conserved residues is above $30 \%$ (Sengupta and Chandra, 2010). The 3-D structure, when analyzed with Ramachandran plot, showed $87.2 \%$ residues in the most favored region, $11.5 \%$ in the additionally allowed region, $1.0 \%$ in generously allowed region, and $0.3 \%$ in disallowed region, which indicated that the model is of good quality. Structural similarity of this model with 1J0X_R when analyzed using the FATCAT software showed that the 2 structures overlapped reasonably well (Figure 5B). The RMSD value of PeGPD against $1 \mathrm{~J} 0 \mathrm{X} \_\mathrm{R}$ is 0.15 , which indicates that the structures are significantly similar, and hence, the structural positions of the catalytic residues can be accurately predicted from the model. When compared to the structure of 1J0X_R (Sirover, 1999; Cowan-Jacob et al., 2003), the following features have been found in PeGPD: Cys ${ }^{151}$ appears to be at the binding site of the substrate glyceraldehyde-3phosphate and plays a role in creating the hemithioacetal intermediate; Lys ${ }^{185}$ is involved in phosphate binding to $\mathrm{NAD}^{+}$; the residues $\mathrm{Phe}^{36}$ and $\mathrm{Phe}^{101}$ interact with the adenine ring, $\mathrm{Asp}^{34}$ with the adenosine ribose, $\mathrm{Gly}^{11}, \mathrm{Arg}^{12}$ and $\mathrm{Ile}^{13}$ with the NAD ${ }^{+}$phosphate, and Gly ${ }^{99}$ and $\mathrm{Ala}^{125}$ with the nicotinamide ribose.
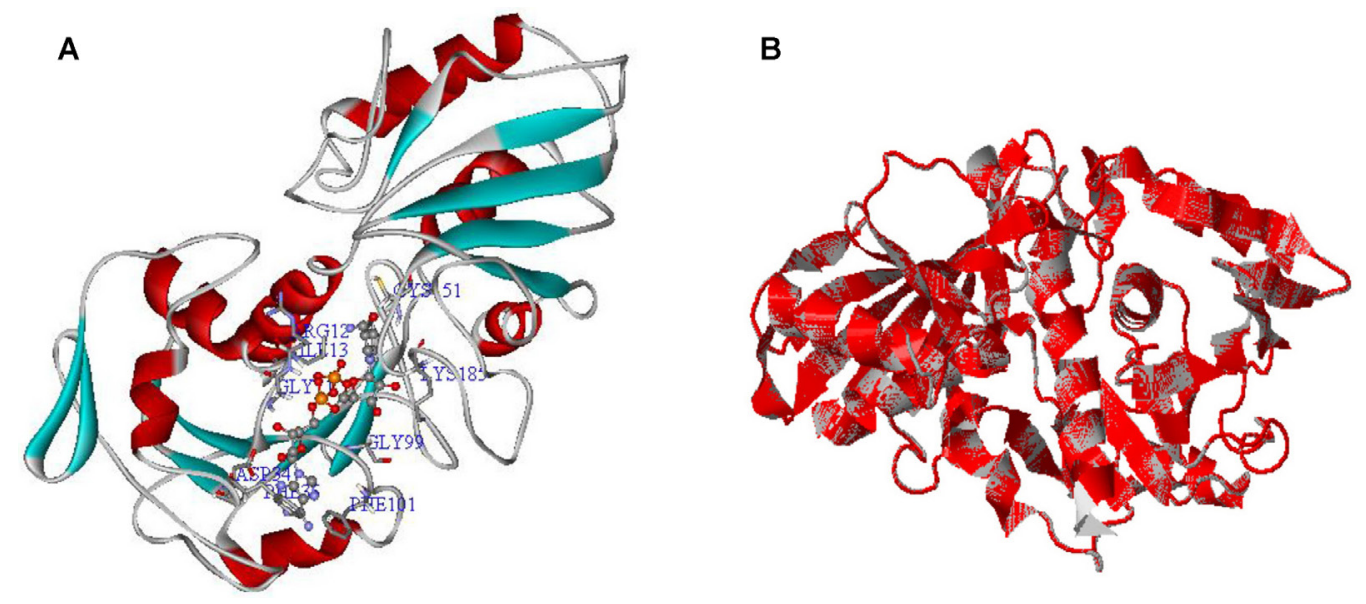

Figure 5. A. Homology model of the PeGPD (ribbon), using the crystal structure of GPD from Oryctolagus cuniculus (PDB: 1J0X_R) as the template. The conserved amino acids were labeled as stick and the substrate NAD as scaled ball and stick. B. Comparison of the predicted structure of PeGPD by superposition of PeGPD (gray) onto the template structure of GPD from O. cuniculus (PDB: 1J0X_R) (red) using FATCAT. 


\section{Southern blot analysis}

The number of genes encoding GPD varies among diverse fungal species. GPD have been cloned as a single-copy gene from R. miehei (Vastag et al., 2004) and Candida bombicola (Van Bogaert et al., 2008). However, GPD were also found to be multicopy genes in M. circinelloides, where 3 GPD copies were isolated (Wolff and Arnau, 2002). To investigate whether $P e G P D$ was a single- or a multiple-copy gene, Southern blot analysis was performed under high-stringency conditions, and one clear band appeared in lanes loaded with genomic DNA samples digested with EcoRI, HindIII, KpnI, DraI, and BamHI (Figure 6), which indicated a single copy of the GPD gene in the genome of $P$. expansum PE-12, and consequently, it is plausible that the cloned gene is functional.

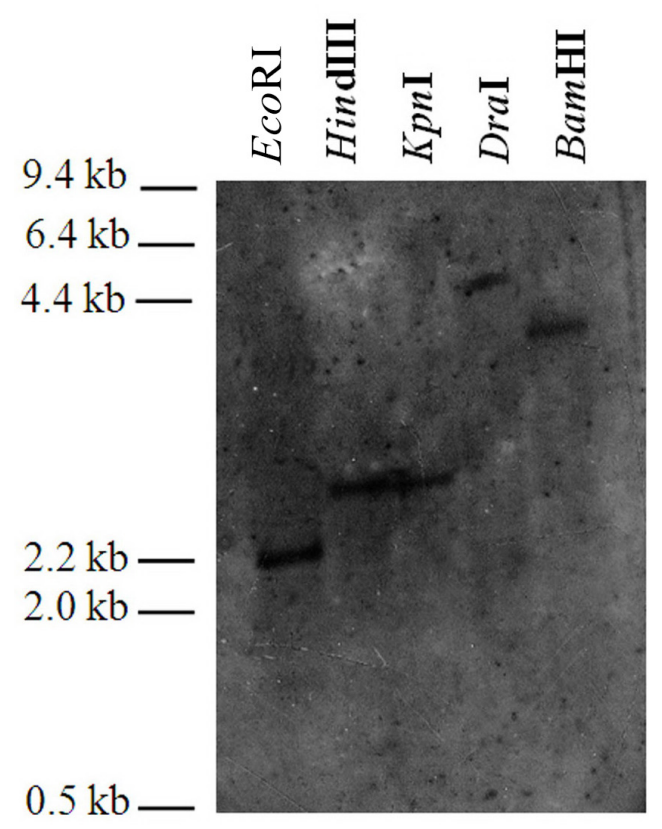

Figure 6. Southern blot analysis of GPD in Penicillium expansum. Total genomic DNA isolated from fresh mycelium of $P$. expansum PE-12 was digested overnight at $37^{\circ} \mathrm{C}$ with EcoRI, HindIII, KpnI, DraI, BamHI, followed by hybridization with the alkaline phosphatase-labeled $P e G P D$ probe.

\section{Gene expression analysis}

The transcriptional strength of $P e G P D$ and $P E L$ was evaluated by semi-quantitative RT-PCR. As a result (Figure 7), the expression level of $P e G P D$ was much higher than that of $P E L$. Combined with the Southern blot results, we can conclude that the highly expressed $P e G P D$ was not due to its gene dosage but to its own highly active promoter. Therefore, we will further investigate the corresponding cis-regulatory elements of $P e G P D$. Further cloning the promoter regions of $P e G P D$ gene will promote the over-production of PEL through homologous expression in $P$. expansum PE-12. 


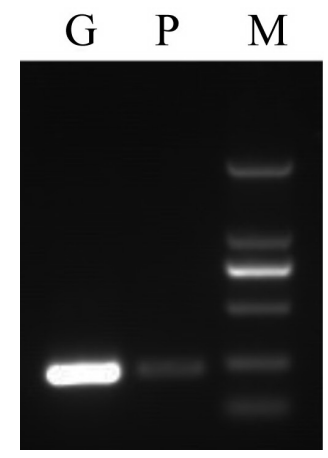

Figure 7. Expression analysis of GPD in Penicillium expansum. Total RNA (400 ng) was isolated from $P$. expansum PE-12 and subjected to RT-PCR amplification. Lane $G=$ RT-PCR amplification for $G P D$; lane $P=$ RTPCR amplification for PEL; lane $M=$ DL-2000 marker (Takara). Bands from top to bottom: 2000, 1000, 750, 500, 250 , and $100 \mathrm{bp}$, respectively.

This paper reports the isolation and characterization of the gene coding for GPD of $P$. expansum. In expectation of a high and constitutive expression, the promoters of GPD genes have been used in various transformation vectors of different fungi (de Groot et al., 1998), among which heterologous promoters and regulatory elements are reported in several successful cases (Zhong et al., 2007; Zhang et al., 2008), but in most cases, homologous ones have been found to be more efficient (Ma et al., 2003; Kajita et al., 2004; Sugiura et al., 2009). The data presented here and the availability of the homologous expression signals of the $P e G P D$ allow the construction of similar transformation vectors, which should extend our understanding of this industrially important fungus.

\section{ACKNOWLEDGMENTS}

Research supported by the China National High-Tech " 863 " Program, the China Ministry of Education and the Shanghai Leading Academic Discipline Project.

\section{REFERENCES}

Bian C, Yuan C, Lin L, Lin J, et al. (2005). Purification and preliminary crystallographic analysis of a Penicillium expansum lipase. Biochim. Biophys. Acta 1752: 99-102.

Cowan-Jacob SW, Kaufmann M, Anselmo AN, Stark W, et al. (2003). Structure of rabbit-muscle glyceraldehyde-3phosphate dehydrogenase. Acta Crystallogr. D Biol. Crystallogr. 59: 2218-2227.

Dai D and Xia L (2005). Enhanced production of Penicillium expansum PED-03 lipase through control of culture conditions and application of the crude enzyme in kinetic resolution of racemic Allethrolone. Biotechnol. Prog. 21: 1165-1168.

de Groot MJ, Bundock P, Hooykaas PJ and Beijersbergen AG (1998). Agrobacterium tumefaciens-mediated transformation of filamentous fungi. Nat. Biotechnol. 16: 839-842.

Fei X, Zhao MW and Li YX (2006). Cloning and sequence analysis of a glyceraldehyde-3-phosphate dehydrogenase gene from Ganoderma lucidum. J. Microbiol. 44: 515-522.

Gustafsson C, Govindarajan S and Minshull J (2004). Codon bias and heterologous protein expression. Trends Biotechnol. 22: $346-353$

Holland MJ and Holland JP (1978). Isolation and identification of yeast messenger ribonucleic acids coding for enolase, glyceraldehyde-3-phosphate dehydrogenase, and phosphoglycerate kinase. Biochemistry 17: 4900-4907. 
Kajita S, Sugawara S, Miyazaki Y, Nakamura M, et al. (2004). Overproduction of recombinant laccase using a homologous expression system in Coriolus versicolor. Appl. Microbiol. Biotechnol. 66: 194-199.

Kumar S, Tamura K and Nei M (2004). MEGA3: Integrated software for Molecular Evolutionary Genetics Analysis and sequence alignment. Brief. Bioinform. 5: 150-163.

Laskowski RA, MacArthur MW, Moss DS and Thornton JM (1993). PROCHECK: a program to check the stereochemical quality of protein structures. J. Appl. Cryst. 26: 283-291.

Li B, Rotsaert FA, Gold MH and Renganathan V (2000). Homologous expression of recombinant cellobiose dehydrogenase in Phanerochaete chrysosporium. Biochem. Biophys. Res. Commun. 270: 141-146.

Ma B, Mayfield MB and Gold MH (2003). Homologous expression of Phanerochaete chrysosporium manganese peroxidase, using bialaphos resistance as a dominant selectable marker. Curr. Genet. 43: 407-414.

Malloch D (1985). The Trichomaceae: Relationships With Other Ascomycetes. In: Advances in Penicillium and Aspergillus Systematics (Samson RA and Pitt JI, eds.). Plenum Press, New York, 365-382.

Piechaczyk M, Blanchard JM, Marty L, Dani C, et al. (1984). Post-transcriptional regulation of glyceraldehyde-3phosphate-dehydrogenase gene expression in rat tissues. Nucleic Acids Res. 12: 6951-6963.

Saitou N and Nei M (1987). The neighbor-joining method: a new method for reconstructing phylogenetic trees. Mol. Biol. Evol. 4: 406-425.

Sambrook J and Russell DW (2001). Molecular Cloning. 3rd edn. Cold Spring Harbor Laboratory, New York.

Sengupta S and Chandra TS (2010). Molecular and structural characterization of GTP-cyclohydrolase II in Eremothecium ashbyi NRRL Y-1363: cDNA cloning, comparative sequence analysis and molecular modeling. Fungal Biol. 114: 731-738.

Sengupta S and Chandra TS (2011). Sequence analysis and structural characterization of a glyceraldehyde-3-phosphate dehydrogenase gene from the phytopathogenic fungus Eremothecium ashbyi. Mycopathologia 171: 123-131.

Sirover MA (1999). New insights into an old protein: the functional diversity of mammalian glyceraldehyde-3-phosphate dehydrogenase. Biochim. Biophys. Acta 1432: 159-184.

Stocklein W, Sztajer H, Menge U and Schmid RD (1993). Purification and properties of a lipase from Penicillium expansum. Biochim. Biophys. Acta 1168: 181-189.

Sugiura T, Yamagishi K, Kimura T, Nishida T, et al. (2009). Cloning and homologous expression of novel lignin peroxidase genes in the white-rot fungus Phanerochaete sordida YK-624. Biosci. Biotechnol. Biochem. 73: 1793-1798.

Thirach S, Cooper CR Jr and Vanittanakom N (2008). Molecular analysis of the Penicillium marneffei glyceraldehyde3-phosphate dehydrogenase-encoding gene $(g p d A)$ and differential expression of $g p d A$ and the isocitrate lyaseencoding gene $(a c u D)$ upon internalization by murine macrophages. J. Med. Microbiol. 57: 1322-1328.

Van Bogaert IN, De Maeseneire SL, Develter D, Soetaert W, et al. (2008). Cloning and characterisation of the glyceraldehyde 3-phosphate dehydrogenase gene of Candida bombicola and use of its promoter. J. Ind. Microbiol. Biotechnol. 35: 1085-1092.

van den Berg MA, Albang R, Albermann K, Badger JH, et al. (2008). Genome sequencing and analysis of the filamentous fungus Penicillium chrysogenum. Nat. Biotechnol. 26: 1161-1168.

Vastag M, Kasza Z, Acs K, Papp T, et al. (2004). Cloning and sequence analysis of the glyceraldehyde-3-phosphate dehydrogenase gene from the zygomycetes fungus Rhizomucor miehei. Antonie Van Leeuwenhoek 86: 111-119.

Wolff AM and Arnau J (2002). Cloning of glyceraldehyde-3-phosphate dehydrogenase-encoding genes in Mucor circinelloides (Syn. racemosus) and use of the gpd1 promoter for recombinant protein production. Fungal. Genet. Biol. 35: 21-29.

Ye Y and Godzik A (2003). Flexible structure alignment by chaining aligned fragment pairs allowing twists. Bioinformatics. (Suppl 2) 19: ii246-ii255.

Yuan C, Lin L, Shi QQ and Wu SG (2003). Overexpression of Penicillium expansum lipase gene in Pichia pastoris. Sheng Wu Gong Cheng Xue Bao 19: 231-235.

Zhang WX, Jiang YM, Zou XL, Chen B, et al. (2001). Breeding of alkaline lipase overproducing strain by screening resistant mutant. Chin. J. Ind. Microbiol. 31: 14-16.

Zhang P, Xu B, Wang Y, Li Y, et al. (2008). Agrobacterium tumefaciens-mediated transformation as a tool for insertional mutagenesis in the fungus Penicillium marneffei. Mycol. Res. 112: 943-949.

Zhang Z, Yang J, Xu L, Liu Y, et al. (2010). Cloning, codon optimization and expression of mature lipase gene Penicillium expansum. Wei Sheng Wu Xue Bao 50: 228-235.

Zhong YH, Wang XL, Wang TH and Jiang Q (2007). Agrobacterium-mediated transformation (AMT) of Trichoderma reesei as an efficient tool for random insertional mutagenesis. Appl. Microbiol. Biotechnol. 73: 1348-1354. 